\title{
Jeu de rôle de table 2.0 : évolution d'une pratique ludique à l'ère du numérique
}

\section{Ugo Roux}

\section{(2) OpenEdition}

\section{Journals}

Édition électronique

URL : http://journals.openedition.org/sdj/741

DOI : $10.4000 /$ sdj.741

ISSN : 2269-2657

\section{Éditeur}

Laboratoire EXPERICE - Centre de Recherche Interuniversitaire Expérience Ressources Culturelles Education

\section{Référence électronique}

Ugo Roux, « Jeu de rôle de table 2.0 : évolution d'une pratique ludique à l'ère du numérique », Sciences du jeu [En ligne], 6 | 2016, mis en ligne le 07 novembre 2016, consulté le 19 avril 2019. URL : http:// journals.openedition.org/sdj/741 ; DOI : 10.4000/sdj.741

Ce document a été généré automatiquement le 19 avril 2019.

Tous droits réservés 


\title{
Jeu de rôle de table 2.0 : évolution d'une pratique ludique à l'ère $d u$ numérique
}

\author{
Ugo Roux
}

1 Cet article s'intéresse à l'évolution de la pratique du jeu de rôle de table à l'ère du numérique et des technologies de l'information et de la communication, principalement en France. Internet, le Web 2.0, et les autres dispositifs numériques sont pratiquement omniprésents dans notre société. Les jeux, notamment les jeux de rôle de table, ne sont pas épargnés par cette imprégnation numérique puisque l'expérience de jeu des « rôlistes » est bien souvent augmentée et améliorée à travers ces nouveaux dispositifs. Ce constat nous amène à nous demander comment les nouveaux comportements et usages liés aux technologies numériques qui ont été adoptés par certains rôlistes - dans le but d'augmenter et d'améliorer leur expérience de jeu - éprouvent, voire redéfinissent, le jeu de rôle de table, que ce soit dans son cadre identitaire ou bien dans sa spatialisation et sa temporalité. Existe-t-il des caractéristiques diagnostiques qui préservent ce qui singularise le jeu de rôle ? Peut-on parler d'essence du jeu de rôle?

2 Pour répondre à cette question, nous adopterons plusieurs angles d'approche, qui se veulent toutefois complémentaires. Nous tenterons dans un premier temps de donner une définition de ce qu'est le jeu de rôle de table. « Donner une définition claire et précise du jeu de rôles n'est pas chose facile» (Dauphragne, 2011a, p. 195). Effectivement, la complexité de cette forme de jeu empêche d'en donner une définition succincte qui ne soit pas lacunaire. Il s'agit d'ailleurs d'une question qui fait encore débat chez les rôlistes, ce que l'on peut fréquemment constater sur des fils de discussion en ligne traitant du sujet, que ce soit sur les réseaux sociaux ou sur des forums (la figure 1 présente une discussion à ce propos qui a été initiée par une publication datant du 28 juin 2015 dans le groupe Facebook «Discussions de Rôlistes »; la figure 2 présente l'introduction d'un article datant du 2 février 2016 sur la définition du jeu de rôle publié sur le site «Aux Portes de l'Imaginaire » par Laurent Gärtner). 
Qui donnes selon vous la meilleure définition du jeu de rôle?

If Jaime Commenter $\Rightarrow$ Partager
(1) Vous et 3 autres personnes
Voirles commentaires précédents

Publication dans le groupe Facebook « Discussions de Rôlistes » à propos de la définition du Jeu de Rôle (source : Discussions de Rôlistes (Facebook). URL : http://bit.ly/2cda3MR)

Figure 2

\section{Définir le jeu de rôle}

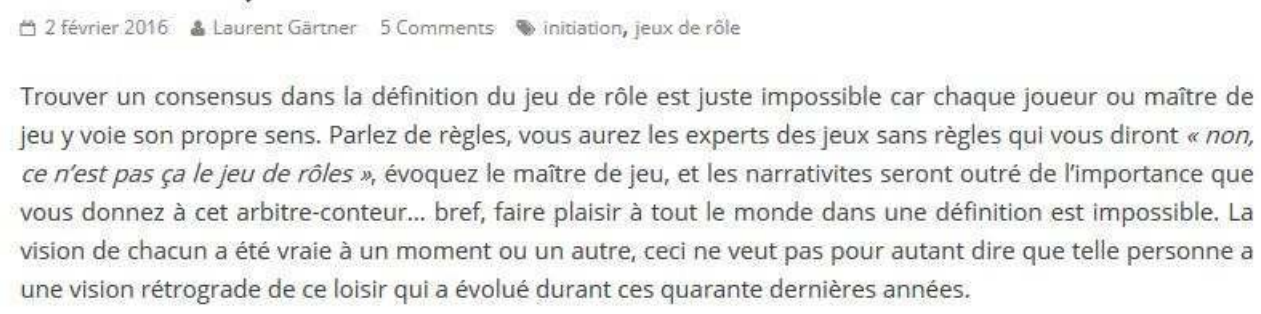
jeu y voie son propre sens. Parlez de règles, vous aurez les experts des jeux sans règles qui vous diront "non, ce n'est pas ça le jeu de rôles ", évoquez le maître de jeu, et les narrativites seront outré de l'importance que vous donnez à cet arbitre-conteur... bref, faire plaisir à tout le monde dans une définition est impossible. La vision de chacun a été vraie à un moment ou un autre, ceci ne veut pas pour autant dire que telle personne a une vision rétrograde de ce loisir qui a évolué durant ces quarante dernières années.

Introduction d'un article sur la définition du jeu de rôle publié sur le site Internet « Aux portes de l'imaginaires » (source : Laurent Gärtner (Aux Portes de l'Imaginaire). URL : http://bit.ly/2cdgNtU)

Ce travail propose dans un deuxième temps d'observer les nouveaux comportements et usages numériques des rôlistes français qui - par le biais de ces comportements et usages - souhaitent augmenter et améliorer leur expérience de jeu lors de parties de jeu de rôle de table. Comme sur ce point l'approche théorique semble limitée sur le plan épistémologique, nous avons opté pour la méthode des questionnaires afin de réaliser cette observation. Pour cela nous avons soumis un questionnaire à de nombreux sujets (rôlistes) afin de récolter des données sur les comportements et usages numériques auxquels nous faisions référence.

\section{Qu'est-ce que le jeu de rôle de table ou papier?}

Il existe aujourd'hui de nombreux jeux de rôle de table ou papier, ${ }^{1}$ qu'ils soient professionnels ou amateurs, le plus connu d'entre eux étant certainement Donjons et Dragons (Dungeons \& Dragons en anglais, aussi connu sous l'acronyme D\&D). Ce « nouveau format ludique » (Dauphragne, 2008) créé par l'américain Ernest Gary Gygax, coécrit avec David (Dave) Arneson et copublié avec Don R. Kaye sous licence Tactical Studies Rules (TSR), et sorti pour la première fois en 1974. Si le jeu de rôle - entendu comme l'interprétation d'un personnage, dans des logiques de mimicry (Peyron, 2008) - existait sous des formes « simples » (comme par exemple des enfants jouant aux cow-boys et aux indiens) avant cette date (Fine, 2002), Gygax et Arneson sont les premiers à le codifier 
sous forme de manuel renfermant des règles. Cette initiative découle d'une volonté de faire évoluer une forme de jeu existant, les wargames, et en particulier Chainmail développé en 1971 par Gary Gygax et Jeff Perren. L'enjeu était d'impliquer d'avantage le joueur et de renforcer son immersion dans le jeu et l'univers présenté, par le biais d'une projection identitaire (Peyron, 2008), en lui proposant d'interpréter une individualité qui serait son avatar, ou alter ego, en jeu et de lui faire vivre de nombreuses aventures, quêtes, et autres missions. Le joueur, que l'on nomme rôliste, aurait alors la possibilité de développer la personnalité et la psychologie de son personnage, ses capacités physiques et mentales, et bien d'autres aspects encore.

Le jeu de rôle de table est une forme de jeu de simulation qui demande à ses joueurs de développer une fiction ludique. Par conséquent, ce jeu propose une «forme particulière de jeu de société reposant sur l'imagination des joueurs et sur des mécanismes ludiques virtuels » (Dauphragne, 2011d, p. 195). Il s'agit d'un format entre jeu et fiction qui permet au joueur « de s'actualiser dans le cadre du jeu comme s'il n'était pas lui-même mais un autre être vivant dans un autre environnement» (Shulga, 2003, p. 103). En d'autres termes, le joueur interprète un personnage imaginaire - auquel il s'identifie plus ou moins - qui s'inscrit dans le contexte du jeu et qui sera son principal dispositif et vecteur immersif dans l'univers fictionnel. À travers celui-ci, le joueur peut faire évoluer la fiction ludique en agissant par une "immersion mimétique" (Peyron, 2008) qui se veut cohérente avec l'univers en place (e.g. Médiéval-Fantastique, Science-Fiction, Space Opera, Cyberpunk). ${ }^{2}$ En d'autres termes, le rôliste - qui évolue dans un large espace d'appropriation - peut agir virtuellement selon ses désirs dans les limites imposées par différents paramètres (propres au jeu et à l'univers de jeu), qu'ils soient permanents, temporaires ou contextuels (ceux-ci peuvent être, entre autres, psychologiques, physiques, environnementaux, comportementaux, raciaux). Ces paramètres seront rappelés, si besoin est, par le maître du jeu qui a la gestion de tout l'univers extérieur aux personnages des joueurs et de sa cohérence. Selon Dauphragne (2011c), il ne peut donc y avoir de jeu de rôle de table sans que le rôliste n'accepte l'immersion fictionnelle puisque l'essence du jeu est l'exploration imaginaire.

Autrement dit, une partie de jeu de rôles consiste aussi bien en une construction fictive collective qu'en une dynamique ludique évolutive qui permet aux joueurs une totale liberté d'action et de décision, dans les limites de la vraisemblance de l'univers fictif dans lequel se déroule le scénario. (Dauphragne, 2011d, p. 195).

6 Le jeu de rôle papier est un jeu que l'on pourrait qualifier de collaboratif et de coopératif dans la mesure où les joueurs doivent participer - à travers les personnages qu'ils interprètent - au déroulement d'une fiction ludique dont l'enjeu est l'atteinte d'un ou plusieurs objectifs qui ne sont pas nécessairement clairement fixés au début du jeu. Cette contribution plurielle - de la part des joueurs et du meneur - à la création de l'histoire se fait en respectant une convention d'intervention plus ou moins définie. Dauphragne (2008) qualifie cela de "récit à plusieurs voix ». En effet, « le format narratif d'action est une caractéristique spécifique des jeux de rôles papier » (Shulga, 2003, p. 106) puisque les joueurs n'agissent pas physiquement lors d'une partie de jeu de rôle de table (à l'inverse des jeux de rôle grandeur nature); seule l'action virtuelle verbalisée (généralement oralisée) des joueurs permet aux personnages d'interagir entre eux et avec l'univers de jeu. La fiction ludique développée lors d'une partie de jeu de rôle de table est déclinée sous la forme d'une trame - plus ou moins complexe et plus ou moins directive - qui est proposée par un maître du jeu (MJ, meneur de jeu, ou encore, selon les terminologies propres aux divers jeux de rôle, maître du donjon (Donjons \& Dragons), gardien des arcanes 
(l'Appel de Cthulhu)...). Cette trame prend généralement la forme d'un canevas (ou scénario) ${ }^{3}$ ou d'une campagne, entre autres, qui présente une situation initiale qui se développe avec les actions des joueurs. Elle est le « cœur » du jeu de rôle papier puisque le jeu ne prend tout son sens que s'il est rapporté à un récit, un contexte imaginaire, un univers de fiction ${ }^{4}$ (Dauphragne, 2011a, 2011c).

Ce sont les interactions entre joueurs et maître de jeu qui vont constituer la trame $\mathrm{du}$ jeu, la situation ludique en elle-même ; à cet égard le contexte du jeu, c'est-àdire l'univers de fiction dans lequel évoluent les rôlistes, a autant d'importance que l'ensemble des règles qui permettent aux joueurs d'agir dans cet univers. Le monde imaginaire et la mécanique ludique apparaissent ainsi comme les deux piliers d'une partie de jeu de rôle (Dauphragne, 2009, p. 70).

\section{Le numérique comme nouvelle façon de penser le jeu de rôle de table}

7 Notre propos se concentre sur la façon dont les technologies de l'information et de la communication « enrichissent » le jeu de rôle de table sans en altérer les caractéristiques diagnostiques. Lorsque l'on fait référence aux jeux de rôle à l'ère du numérique, la première chose à laquelle on pourrait penser sont les jeux vidéo qui sont un "art d'emprunt " (Jolivalt, 1994) au genre traité ici : le jeu de rôle papier. Nous pensons tout particulièrement à deux types de jeux vidéo : le MUD (Multiple User Dungeon) et le RPG ( Role Playing Game) ainsi que leurs déclinaisons. Ces adaptations vidéoludiques des jeux de rôle ne peuvent cependant pas être considérées comme "de table » ou "papier » et ce, pour deux raisons majeures. Nous soutenons la thèse de deux conditions essentielles concomitantes pour identifier un jeu de rôle de table: 1 . la possibilité du jeu de s'affranchir de tout dispositif et de toute ressource ${ }^{5}$ extérieurs aux rôlistes pour potentiellement se reposer exclusivement sur leur cognition afin de "construire» l'univers de jeu (la mémoire ou l'imagination peuvent notamment se substituer aux manuels de règles) ; 2 . la présence nécessaire d'une médiation humaine directe entre les joueurs et l'univers de jeu (le maître du jeu fait généralement office de médiateur puisqu'il assure la gestion de tout l'univers extérieur aux personnages des joueurs ainsi que sa cohérence). Le corollaire de ces conditions fait du jeu de rôle papier un jeu essentiellement collectif puisqu'il nécessite d'être minimum à deux joueurs (dont un maître du jeu) pour créer le cadre du jeu par un échange verbalisé qui est la condition nécessaire pour actualiser le contexte imaginaire (Shulga, 2003).

\section{Tour d'horizon des comportements et des usages des rôlistes à l'ère du numérique}

Le jeu de rôle papier est une activité essentiellement cognitive et l'imaginaire des joueurs en est le "terrain de jeu». Effectivement, "dans les jeux papier, la transmission de l'information sur l'univers de jeu se passe par l'interaction entre les joueurs, car c'est au cours des conversations que cet univers prend une forme et se construit » (Shulga, 2003, p. 110). Comme nous avons pu le voir, à l'inverse du jeu vidéo qui ne peut s'affranchir du dispositif technologique qui crée son identité, le jeu de rôle de table peut, lui, potentiellement s'affranchir de tout dispositif et de toute ressource extérieurs au rôliste. Dauphragne $(2008,2011 b, 2011 c)$ relève également cet aspect fortement dématérialisé du jeu dont la clé réside sur l'imaginaire du joueur. L'humain est le seul «matériel» 
nécessaire et suffisant pour créer et jouer une partie de jeu de rôle papier. Cependant, à l'instar de nombreuses autres activités, le jeu de rôle de table a vu naître de nouveaux comportements et usages de la part de ses joueurs avec le développement des technologies de l'information et de la communication (TIC). Aujourd'hui, les dispositifs et ressources numériques s'invitent fréquemment pour améliorer l'expérience de jeu des rôlistes. L'accès facilité à ces dispositifs et ressources ainsi que leur développement (e.g. technologique, technique, pratique) rendent plus aisée leur intégration dans une partie de jeu de rôle de table. Par conséquent, il est par exemple fréquent de pouvoir observer, entre autres usages récents, des tablettes se substituer au papier pour afficher des aides de jeu, ou encore des logiciels de lancer de dés remplacer les dés traditionnels. Ce travail propose d'observer ces nouveaux comportements et usages.

9 Pour ce faire, nous avons procédé à une enquête par questionnaire de juin 2015 à août 2015. Le questionnaire a été soumis à des rôlistes via des billets explicitant notre recherche et qui ont été postés sur des plateformes socionumériques dédiées à la pratique du jeu de rôle fréquentées par ces individus (e.g. des groupes Facebook comme «Discussions de rôlistes", «RDF Rôliste de France» ou encore "Union Rôliste Francophone", des forums comme "Opale Rôliste» ou celui de la FFJDR, des blogs comme celui de « Kerlaft le rôliste »). Par conséquent, notre cible était des rôlistes portés sur l'utilisation d'Internet et des médias socionumériques (486 individus ont répondu à notre questionnaire). Leurs réponses nous permettent de dégager des tendances que nous présenterons, mais dont le caractère généralisable à l'ensemble de la population rôliste reste à vérifier dans la mesure où celles-ci portent sur l'échantillon particulier qui était le nôtre, à savoir les rôlistes usagers d'Internet et des médias socionumériques. Le questionnaire comprenait 27 variables et fut créé grâce à l'application en ligne du site fr.ze-questionnaire.com qui permet la génération d'un lien exportable qui renvoie audit questionnaire afin de pouvoir y répondre. Nous en livrons les résultats et leur interprétation dans les parties qui suivent.

\section{Détournements et usages numériques}

10 Le jeu de rôle a notamment vu son accès aux ressources (e.g. aides de jeu, scénarios, suppléments de règles) évoluer avec le développement d'Internet. À moins de créer ses propres jeux, à l'époque où les premiers jeux de rôle sont sortis, on ne pouvait se les procurer qu'en se déplaçant en boutique ou en se les prêtant entre amis. Pour ce qui était des aides de jeu, entre autres, il fallait procéder de la même façon. Avec la création des groupes numériques de rôlistes (e.g. forums, réseaux sociaux), des boutiques et des sites en ligne dédiés aux jeux de rôle, l'acquisition des ressources de jeu s'est trouvée facilitée. Ainsi, les créateurs de contenus, professionnels (e.g. éditeurs) comme amateurs, disposent de plateformes en ligne sur lesquelles ils peuvent mettre des contenus à disposition des rôlistes et aussi solliciter leur mobilisation pour la création de contenus (financements participatifs ou crowdfunding).

11 Le jeu de rôle de table en soi ne nécessite que peu de matériel, voire aucun comme nous avons pu le constater. Pour entamer une partie, il suffit, la plupart du temps, du livre de règles pour le maître du jeu, de dés, de papier et de crayons. Il est toutefois possible d'améliorer l'expérience de jeu en rajoutant quelques accessoires (qu'ils soient vestimentaires, décoratifs, ou autres) ainsi que d'autres ressources (comme des aides de jeu par exemple) ou dispositifs. C'est autour de ces deux derniers points que nous 
articulerons notre propos dans cette partie. Avec l'essor des technologies du numérique, les rôlistes ont intégré dans leur pratique des dispositifs numériques dans le but d'améliorer leur expérience de jeu. Au-delà de cet usage d'outils déjà existants, les rôlistes ont également développé des dispositifs pour répondre aux besoins qui leur sont spécifiques. Ces dispositifs se déclinent généralement sous la forme de logiciels facilitant la mise en place de certains éléments et aspects du jeu (e.g. jets de dés, feuilles de personnages).

Une des spécialités des pratiquants de jeux de rôle est le détournement de dispositifs nonludiques vers un usage ludique pour l'amélioration de leur expérience de jeu. Près de la moitié des personnes interrogées (48,6\%) détournent des ressources et/ou des dispositifs numériques qui ne sont pas dédiés aux jeux de rôle pour s'en servir à des fins ludiques (question: "Lors de parties de jeux de rôle, détournez-vous des ressources et/ou dispositifs numériques mais qui ne sont pas dédiés aux jeux de rôle pour améliorer votre expérience de jeu?»). Ces derniers peuvent être de natures très variées. Cela peut aller des simples images sur Google Images aux logiciels et plateformes à but professionnel en tous genres (comme par exemple Realtime Board qui fournit un espace de travail collaboratif en ligne au service des professionnels). Les différents usages ne s'arrêtent pas au détournement de dispositifs et ressources numériques. Effectivement, le hardware fait également l'objet d'un usage pour une large majorité d'individus interrogés $(80,7 \%)$ (figure 5) (questions : «Lors de parties de jeux de rôle, utilisez-vous des dispositifs tels que des smartphones, des tablettes, ou encore des ordinateurs pour améliorer votre expérience de jeu en présentiel ? »; «Si oui, lesquels ?»).

Figure 3

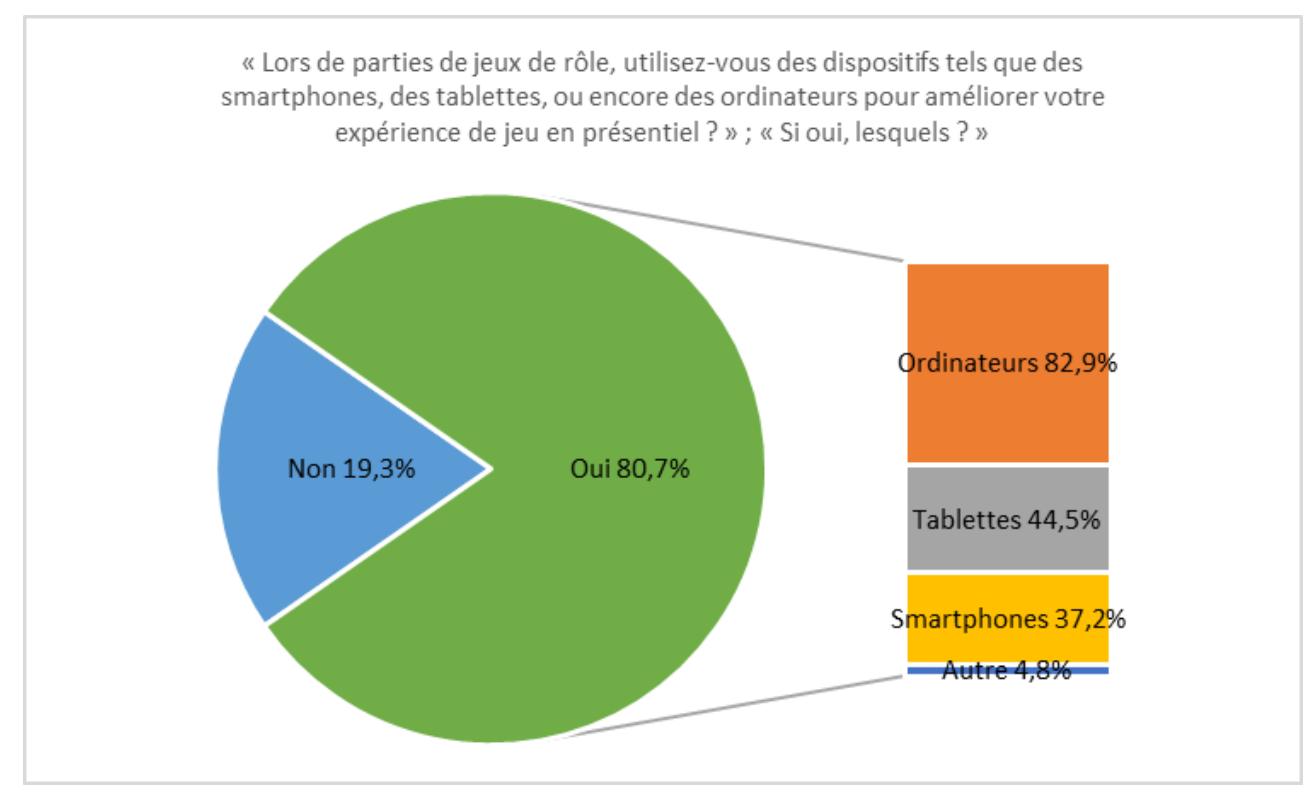

NATURE DES DISPOSITIFS NUMÉRIQUES MATÉRIELS LES PLUS UTILISÉS PAR LES RôLISTES LORS DE LEURS PARTIES DE JDR

13 L'utilisation de ce hardware permet de répondre à divers objectifs dont la finalité est d'améliorer l'expérience de jeu des rôlistes de plusieurs façons (figure 3) (questions: "Lors de parties de jeux de rôle, utilisez-vous des dispositifs tels que des smartphones, des tablettes, ou encore des ordinateurs pour améliorer votre expérience de jeu en présentiel ?»; « Et pour quel(s) usage(s) ? »). Les individus reconnaissent que ces usages 
facilitent nombre d'aspects propres à la pratique du jeu de rôle en elle-même, mais qui lui sont également satellites (figure 5) (question : «Selon vous, les ressources, dispositifs et autres outils précédemment cités facilitent...»). En revanche, pour une petite part des individus questionnés (3,7\%), l'usage de tels dispositifs et ressources n'apporte rien de particulier lors de parties de jeux de rôle.

Figure 4

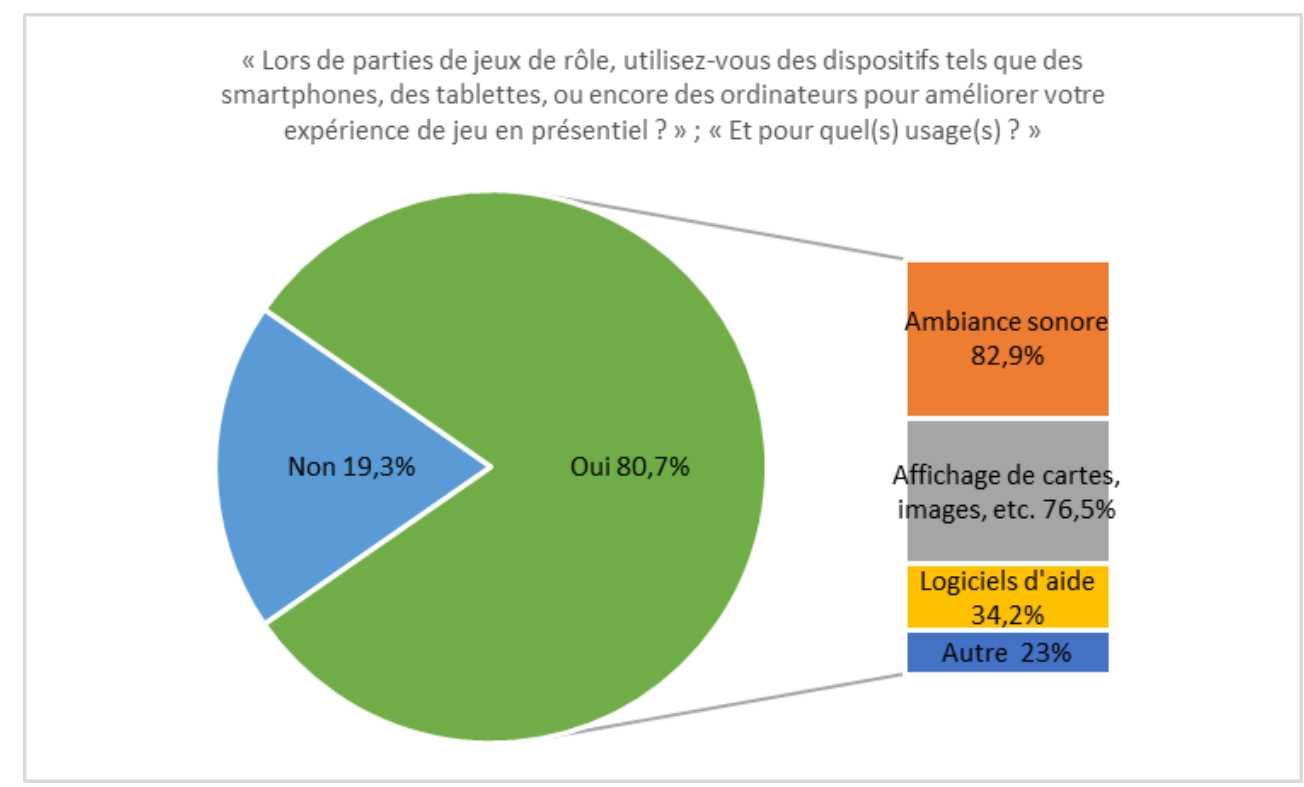

Objectifs les plus recherchés par les rôlistes via l'usage des dispositifs numériques matériels lors de leurs parties de JdR

Figure 5

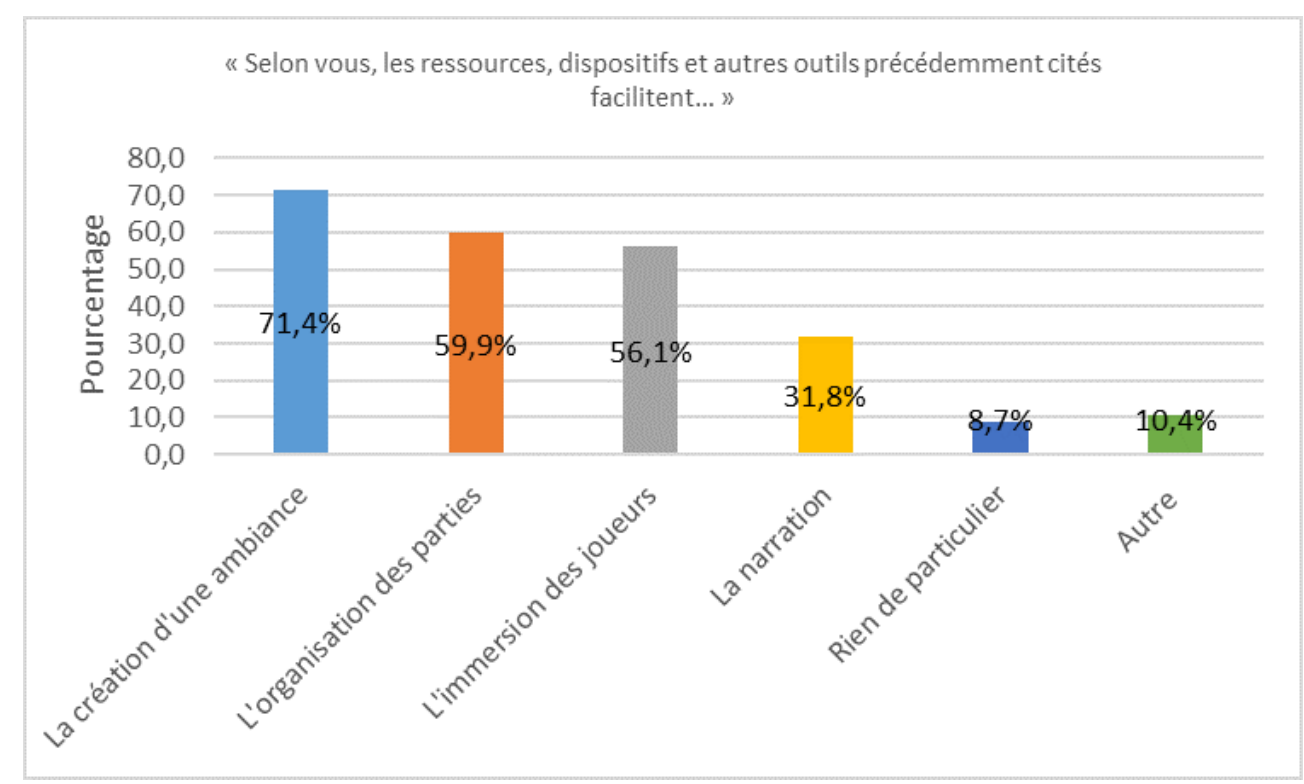

Objectifs remplis et facilités par l'usage de ressources et dispositifs numériques (dématérialisés ou non) par les rôlistes lors de leurs parties de JdR 
14 Si la population rôliste a su intégrer dans sa pratique des dispositifs déjà existants, elle a également su développer des dispositifs dédiés à la pratique « rôlistique » qui répondent aux besoins qui lui sont spécifiques. Il apparaît dans le cadre de notre enquête qu'une très grande majorité des rôlistes interrogés $(89,5 \%)$ déclarent utiliser des ressources et/ou des dispositifs numériques - provenant d'Internet - dédiés aux jeux de rôle pour améliorer leur expérience de jeu; ressources et dispositifs numériques qui peuvent être variés (figure 6) et proviennent majoritairement de sites dédiés aux jeux de rôle (figure 7) (questions: Lors de parties de jeux de rôle, utilisez-vous des ressources et/ou des dispositifs numériques dédiés aux jeux de rôle pour améliorer votre expérience de jeu? ; « Si oui, lesquels?»; «Si oui, quels sont les sites Internet où vous trouvez ces ressources et dispositifs?»). D'ailleurs, de nombreux rôlistes interrogés $(42,6 \%)$ répondent être des créateurs de ressources et de dispositifs dédiés aux jeux de rôle ; une grande partie d'entre eux $(45,6 \%)$ déclarent les mettre à disposition sur Internet (questions : «Êtes-vous, vous-même, créateur de ressources ou de dispositifs dédiés aux jeux de rôle ? »; « Si oui, les mettez-vous à disposition sur Internet ?»).

\section{Figure 6}

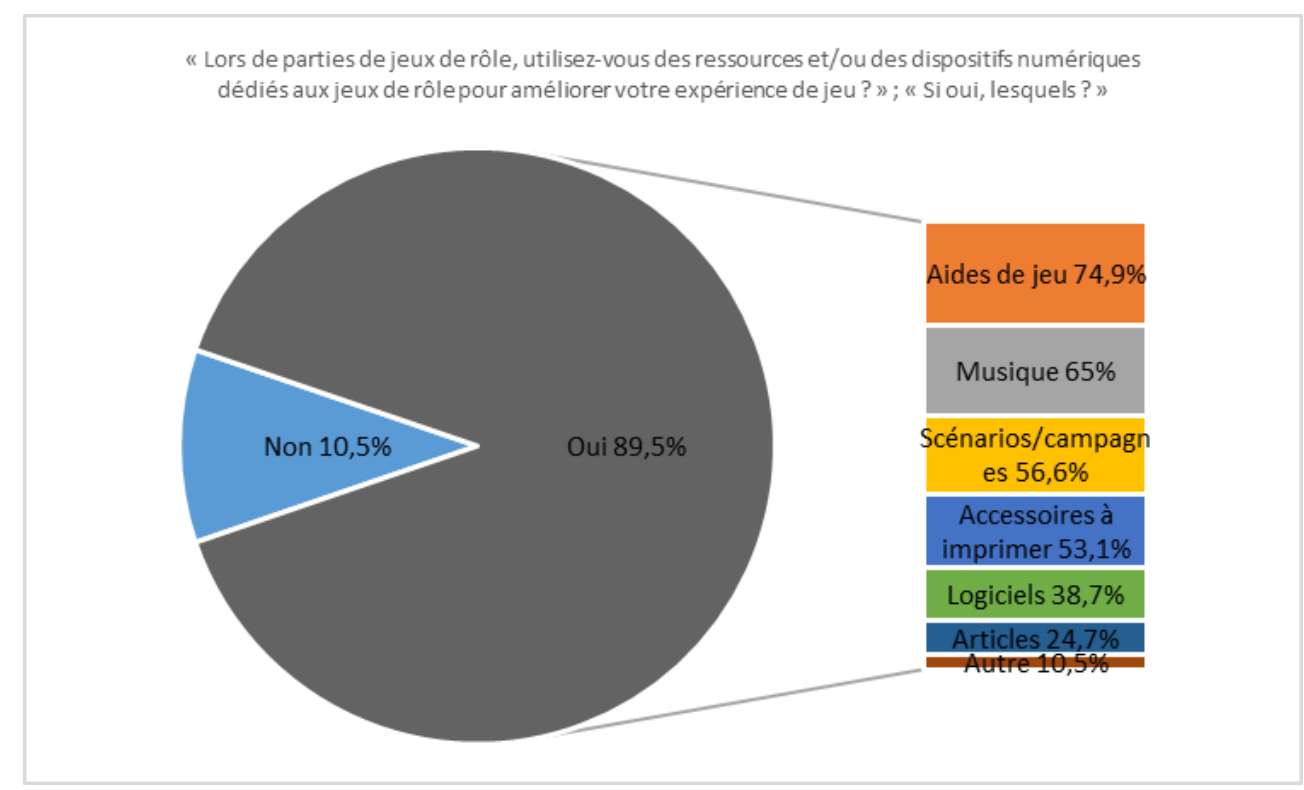

Nature des ressources et dispositifs dédiés aux jeux de rôle les plus utilisés par les rôlistes pour améliorer leur expérience de jeu 


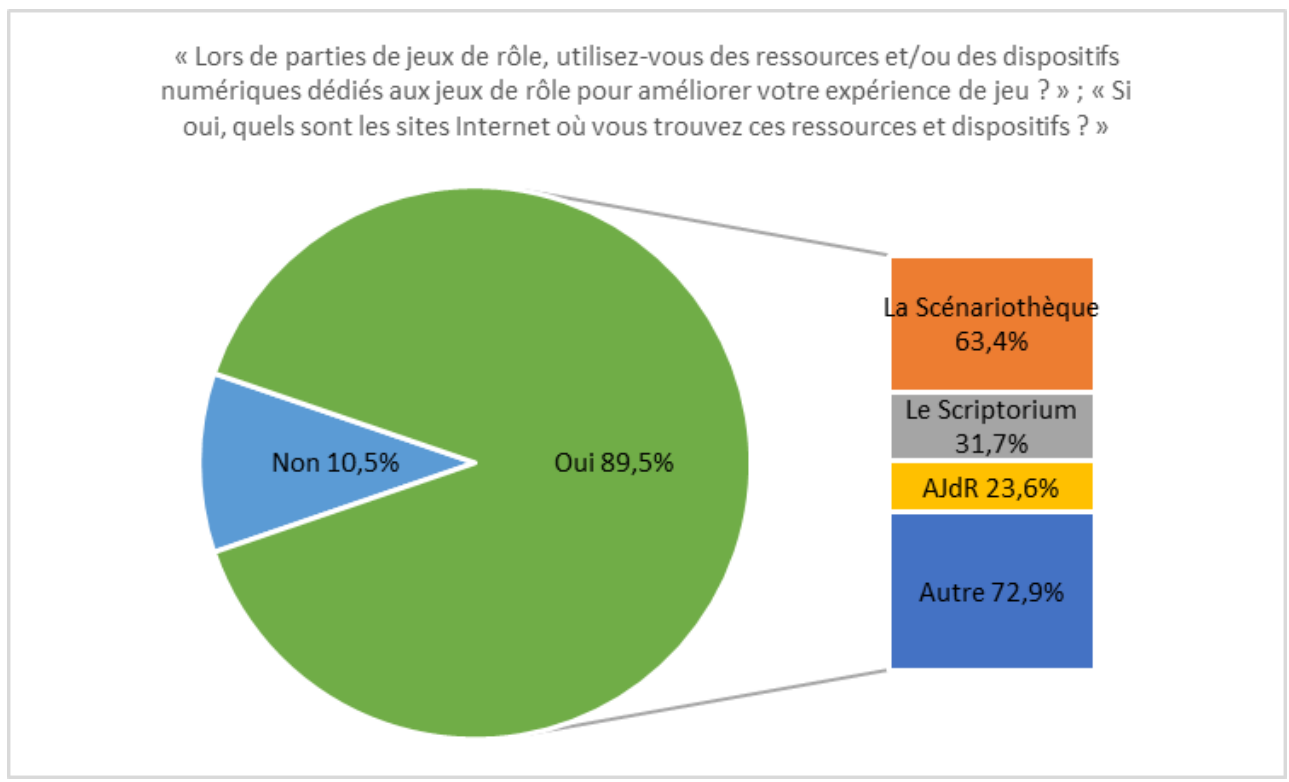

Sources d'acquisition des ressources et dispositifs numériques dédiés aux jeux de rôle

Pour ceux qui n'utilisent aucun des dispositifs, ressources, et autres outils numériques, ceux-là ne le font pas pour diverses raisons. Soit parce qu'ils n'en ont jamais eu l'occasion $(21,2 \%)$, soit parce qu'ils n'ont pas envie d'essayer $(16,6 \%)$, soit parce qu'ils ont déjà essayé et ne veulent plus le refaire $(11,1 \%)$, soit parce que cela manque d'ergonomie $(10,1 \%)$, soit parce que cela n'apporte rien à l'expérience de jeu (12,9\%), soit parce que cela dénature le jeu de rôle (18 \%), soit pour diverses autres raisons (10,1 \%) (question : «Si vous n'utilisez aucun des dispositifs, des ressources ou des outils précédemment cités, pouvez-vous expliciter pourquoi vous ne le faites pas?»).

\section{Dispositifs numériques : vers une redéfinition des frontières spatiales et temporelles du jeu de rôle de table?}

16 Le jeu de rôle de table s'articule autour de plusieurs cadres de participation (Shulga, 2003), qui vont de l'espace commun de communication, au contexte imaginaire de jeu où agissent les personnages. Ces cadres produisent « un espace combiné de présence dans lequel l'interaction des joueurs se déroule et à l'intérieur duquel ils passent constamment d'un plan de présence à un autre " (Shulga, 2003, p. 106). Shulga (2003) distingue trois plans de présence dans les jeux de rôle papier : l'action de jeu qui représente le plan le plus immersif où se déroulent les événements intradiégétiques et où le roleplay ${ }^{6}$ peut se développer; l'action ludique qui représente le plan métafictionnel où la fiction est commentée de façon extradiégétique mais toujours dans un but ludique; le cadre réel où se produisent des événements extérieurs au jeu et donc sans but ludique.

L'un de ces cadres de participation en particulier se voit parfois totalement redéfini avec les technologies de l'information et de la communication. Originellement, l'espace commun de communication des joueurs de jeux de rôle papier se manifeste par la coprésence physique des participants et le partage d'un même espace réel pour assurer le bon déroulement d'une partie. La communication s'opère alors de façon présentielle et 
synchrone. Cependant, l'ère numérique et les technologies de l'information et de la communication modifient la logique de ces cadres de participation. En effet, le développement du Web 2.0, de ses technologies et de ses dispositifs a permis de repenser la pratique du jeu de rôle de table et de redessiner les frontières de ce dernier à la fois dans leur spatialisation et leur temporalité.

Le jeu de rôle papier s'est notamment développé sous une forme "distancielle » qui se veut proche de sa version classique en utilisant des logiciels de VoIP (logiciels de communication orale, e.g. Skype, Mumble, TeamSpeak) permettant une communication synchrone, orale, et souvent visuelle, entre les participants, et ce malgré la distance. Une optimisation de cette pratique réside dans l'emploi de tables virtuelles. Ces logiciels complémentaires, qui peuvent parfois suffire à eux-mêmes s'ils sont dotés d'une fonction VoIP, offrent de nombreux outils pour le bon déroulement d'une partie (e.g. affichage de cartes, jets de dés, images, ambiance sonore).

Un tiers des rôlistes $(32,7 \%)$ interrogés lors de notre enquête répondent jouer sur des "tables virtuelles» dédiées aux jeux de rôle lors de parties à distance (figure 8) (questions : «Utilisez-vous des logiciels ou des plateformes type « table virtuelle » dédiés aux jeux de rôle pour jouer à distance (via Internet)?»; "Si oui, lesquels?»). Pour assurer une communication orale et synchrone lors de leurs parties autour d'une table virtuelle, ces joueurs utilisent des logiciels de VoIP (figure 9) (question : « quel logiciel de VoIP utilisez-vous lors de vos parties de jeux de rôle à distance?»). Le jeu de rôle autour d'une table virtuelle semble être une pratique relativement répandue qui, si elle permet de rassembler des individus géographiquement distants le temps d'une partie, ne satisfait cependant pas totalement les rôlistes. Une très large majorité des rôlistes interrogés (93\%) ne jugent pas l'expérience de jeu à distance équivalente à l'expérience de jeu en présentiel dans la mesure où celle-ci est déficitaire par rapport à certains aspects caractéristiques du jeu en présentiel ; la raison la plus avancée justifiant ce jugement des rôlistes interrogés est notamment le manque d'un «réel » contact humain - par la présence physique des rôlistes - rendant le jeu plus "vivant ", convivial, ou encore immersif, et empêchant ainsi une appréciation totale du jeu (questions: "Jugez-vous l'expérience de jeu à distance (via Internet) équivalente à l'expérience de jeu en présentiel (physiquement) ?»; « Pourquoi ?»). Peut-être pour les raisons précédemment citées, une grande majorité des rôlistes $(77,4 \%)$ répondent jouer plus en présentiel qu'en distanciel tandis que peu font l'inverse (16,1\%), et encore moins (6,5\%) passent autant de temps à jouer en présentiel qu'en distanciel (question: "Quel est votre temps de jeu à distance (via Internet) par rapport à votre temps de jeu en présentiel (physiquement) ?»). L'usage d'une table virtuelle se fait souvent en dernier recours et fréquemment lorsqu'il y a impossibilité de réunir physiquement les joueurs. 
Figure 8

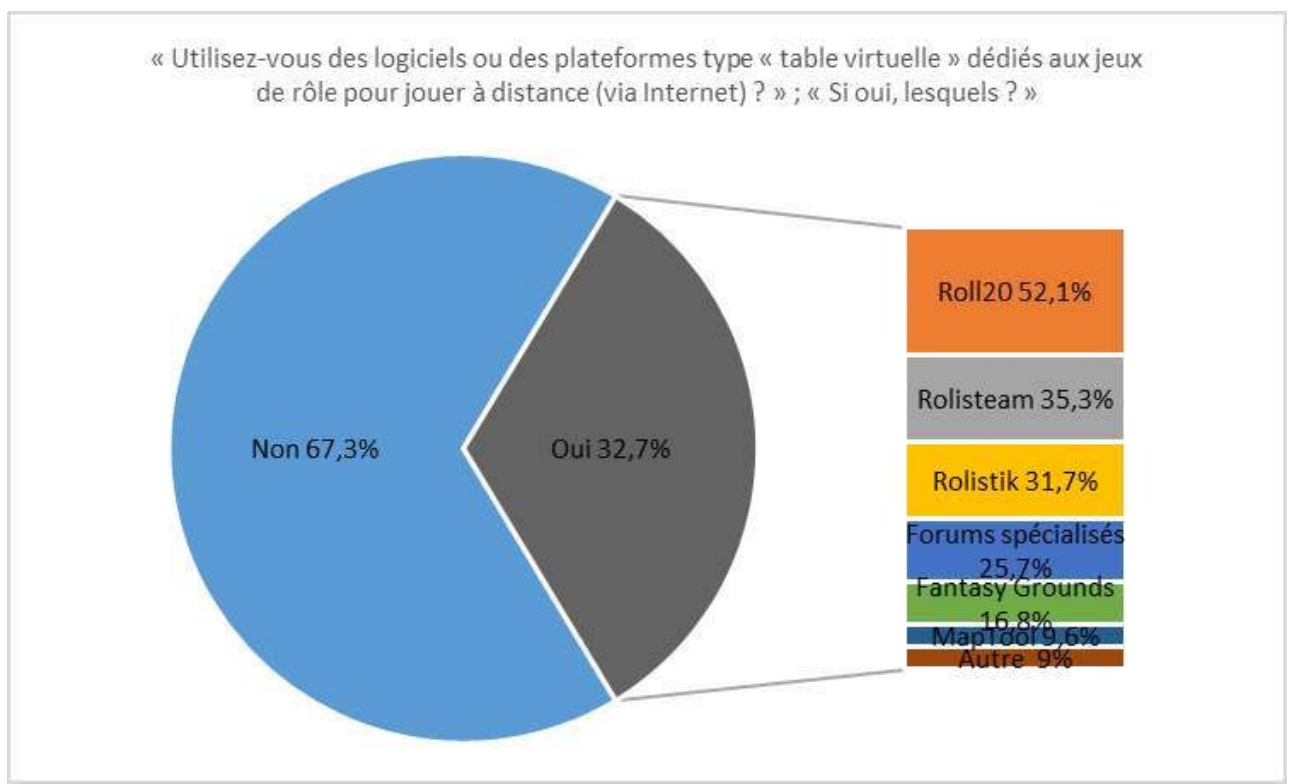

TABles VIRTUelles Les PLUS Utilisées PAR LeS RÔLISTES LORS DE LEURS PARTIES DE JDR EN LIgNE

Figure 9

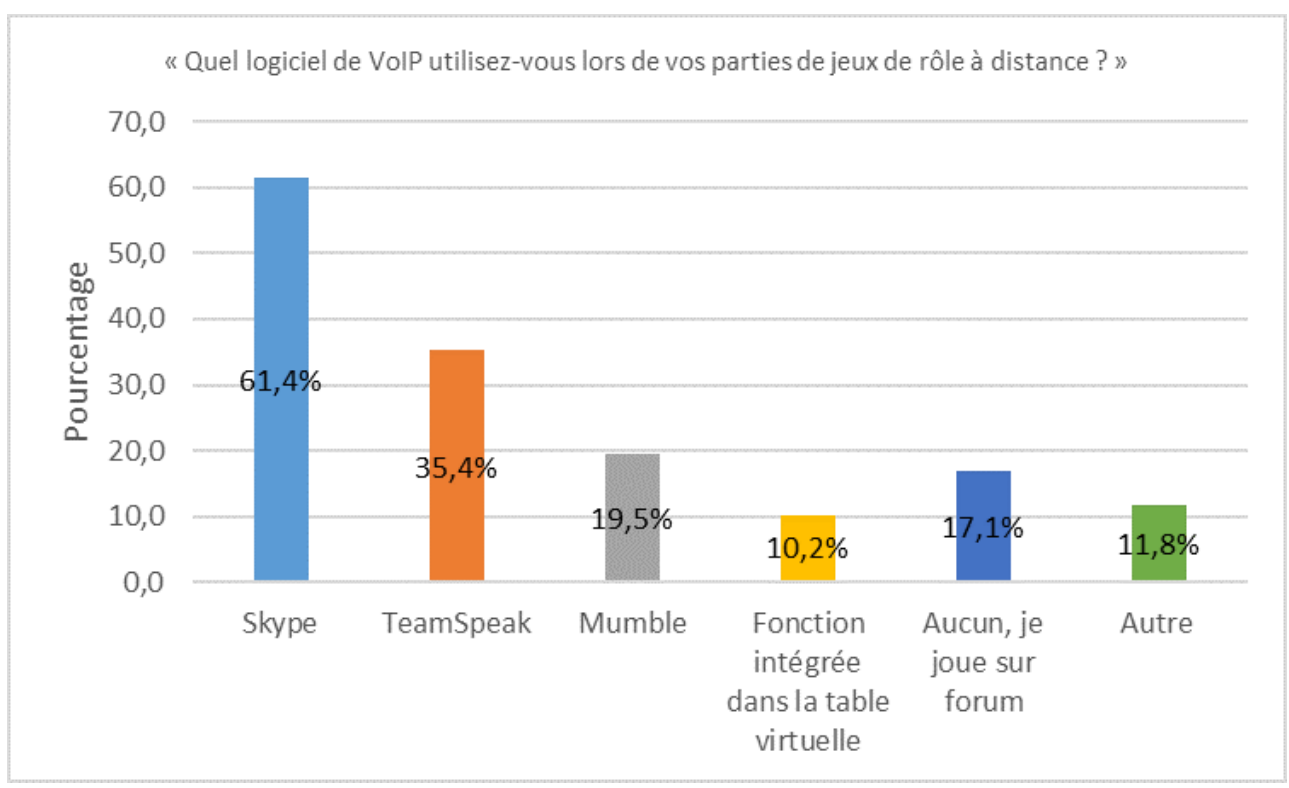

LOgICIELS VOIP LES PLUS UTILISÉS PAR LES RÔLISTES LORS DE LEURS PARTIES DE JDR EN LIgNE

Au-delà de sa spatialisation, le jeu de rôle a également observé une redéfinition de sa temporalité. Effectivement, les technologies de l'information et de la communication ont permis l'émergence de nouvelles formes de jeux de rôle - notamment le jeu de rôle écrit s'articulant autour de moyens de communication asynchrones. Les plateformes socionumériques sont généralement les médias privilégiés de ces nouvelles pratiques.

Le jeu de rôle écrit, ou jeu de rôle textuel ${ }^{7}$ (play-by-post role playing game en anglais), est une pratique qui permet aux rôlistes de participer à un jeu de rôle non seulement en distanciel, mais également sur des temporalités différentes. Il s'agit d'une forme de jeu de 
rôle où l'emphase est particulièrement mise sur les qualités littéraires des participants puisqu'il n'est pas oralisé. Ses origines remonteraient, selon le fondateur du site Internet ptgptb.org Steve Darlington, aux années 1980 (cf. webographie). Cette nouvelle façon de jouer aux jeux de rôle se serait particulièrement développée dans les années 2000 avec l'expansion du Web 2.0 et la simplification des interactions interindividuelles en ligne. Le jeu de rôle écrit se pratique sur des dispositifs socionumériques variés, qui permettent une communication à la fois distante et asynchrone. Cela se traduit par une simplification pratique et logistique dans la mise en place du jeu. La pratique la plus courante de jeu de rôle écrit est celle sur forums spécialisés, mais il en existe de nombreuses autres formes. La pratique sur réseaux sociaux (e.g. Facebook, Twitter, Tumblr) nécessite la création de profils utilisateurs qui sont propres aux personnages interprétés. Ce profil sert à la fois de feuille de personnage mais également de médium avec les usagers du site. Il existe aussi le jeu de rôle par blogs et micro-blogs, par wikis, par emails, ou encore par Google documents. Le jeu de rôle écrit dépasse même les frontières d'Internet pour se pratiquer via SMS. Chacune de ces plateformes offrent des possibilités différentes, plus ou moins complexes, que ce soit dans la mise en place du jeu, son déroulement, ou bien l'immersion des pratiquants.

\section{Conclusion}

Avec ces nouvelles approches et ces nouvelles façons de penser le jeu de rôle, celui-ci s'inscrit dans une logique transmédia de plus en plus marquée. Transmédialité qui était d'ailleurs déjà développée et continuera de l'être avec, entre autres, l'industrie cinématographique, vidéoludique, et littéraire. Un des enjeux majeurs de la narration transmédia des jeux de rôle est le besoin de cohérence dans l'établissement d'un univers. En effet, si le jeu de rôle offre des possibilités narratives quasi-infinies, ce foisonnement créatif se trouve confronté à la nécessité d'une convergence référentielle collective ; convergence que la transmédialité crée en permettant une intertextualité et une acquisition des références et des codes nécessaires à l'appréhension d'un univers donné (Peyron, 2008). D'ailleurs, Peyron (2008) dira du jeu de rôle qu'il est un point de convergence et qu'il est au « carrefour des univers ». Le jeu de rôle présente « une logique de jeu qui transcende les supports» (Dauphragne, 2011a, p. 27). La culture ludique est déjà en soi « une «culture de la convergence » en ce qu'elle repose sur la mobilisation et l'articulation d'une pluralité de médias ; mais c'est aussi une « culture participative », par la place qu'elle accorde aux publics-cibles dans les processus de création et d'évolution de ses mondes imaginaires. [...] le développement de la narration est assuré conjointement par une pluralité de sources. » (Dauphragne, 2011c).

Concernant l'évolution des usages que nous avons étudiés, ces nouvelles pratiques ne remplacent pas les anciennes, bien sûr, mais offrent une approche différente et parfois complémentaire. Internet a notamment fait tomber certaines des barrières spatiales et temporelles qui pouvaient entraver la pratique du jeu de rôle en elle-même d'une part, mais qui pouvaient d'autre part entraver des activités qui lui sont satellites (comme par exemple les recherches documentaires ou bien l'acquisition de contenus). Si de l'aveu de nombreux rôlistes cela facilite bien des aspects de leur pratique du jeu de rôle papier, rappelons toutefois que selon notre enquête, 18 \% des rôlistes parmi ceux qui se refusent d'utiliser des dispositifs et des ressources numériques ne le font pas parce qu'ils jugent que ceux-ci dénaturent le jeu de rôle de table. Cet état de fait pose le problème des limites 
de l'intégration du numérique dans le jeu de rôle. Jusqu'où le numérique peut-il s'immiscer dans cette pratique ludique sans que celui-ci n'altère le jeu de rôle papier dans son identité ? Nous avons défendu la thèse d'un certain nombre d'invariants préservant l'intégrité de l'identité du jeu de rôle de table, notamment la capacité du jeu à s'affranchir de tout dispositif et de toute ressource matérielle, mais surtout la relation humainhumain entre les rôlistes et le médiateur (généralement un maître du jeu) qui a pour tâche de gérer tout l'univers extérieur aux personnages et sa cohérence.

\section{BIBLIOGRAPHIE}

DARLINGTON S., «Une Histoire du jeu de rôle - septième partie : de nouvelles manières de jouer », [en ligne], consulté le 7 septembre 2016, URL : http://ptgptb.fr/une-histoire-du-jdr-7-denouvelles-manieres-de-jouer. Article original : DARLINGTON S., " The History of RolePlaying », [en ligne], consulté le 7 septembre 2016, URL : http://ptgptb.org/0007/hist7.html.

DAUPHRAGNE A. (2008), « Dynamiques ludiques et logiques de genre : les univers de fantasy », in Brougère G. (dir.), La ronde des jeux et des jouets. Harry, Pikachu, Superman et les autres, Paris, Autrement, pp. 43-58.

DAUPHRAGNE A. (2009), « Le Moyen Âge dans les jeux de rôles : simulations ludiques et matière historique », in Abiker S., Besson A., et Plet-Nicolas F. (dir.), Le Moyen Âge en jeu, Actes du colloque international Le Moyen Âge en jeu organisé à Bordeaux du 3 au 5 avril 2008, Eidôlon, 86, Presses Universitaires de Bordeaux, pp. 69-80.

DAUPHRAGNE A. (2011a), « La fantasy au sein de la culture ludique : quand la fiction se donne à jouer », Lecture Jeune, 138, pp. 26-30.

DAUPHRAGNE A. (2011b), « Le jeu de rôles et la Terre du Milieu », in Devaux M., Ferre V., et Ridoux C. (dir.), Tolkien aujourd'hui, Actes du colloque international Tolkien aujourd'hui organisé à Rambures en juin 2008, Presses Universitaires de Valenciennes, pp. 227-239.

DAUPHRAGNE A. (2011c), « Le sens de la fiction ludique : jeu, récit et effet de monde », Strenæ [En ligne], 2, mis en ligne le 21 juin 2011, consulté le 15 août 2016. URL : http:// strenae.revues.org/312.

DAUPHRAGNE A. (2011d), « Les créations moyenâgeuses en littérature de jeunesse et en jeu de rôles : stéréotypes, métissages, innovations » in Cazanave C., et Houssais Y. (dir.), Médiévalités enfantines. Du passé défini au passé indéfini, Besançon, Presses Universitaires de Franche-Comté, pp. 193-212.

DE CANTELOUBE X. (2015), « Le scénario de jeu de rôle : changer de paradigme et replacer les personnages au cœur de l'action », Journées d'études « Les Quarante ans du Jeu de Rôle », du vendredi 12 au dimanche 14 juin 2015, universités Paris 3 et Paris 13.

FINE G. A. (2002), Shared Fantasy: Role-Playing Games as Social Worlds [1983], Chicago et Londres, The University of Chicago Press.

JOLIVALT B. (1994), Les jeux vidéo, Paris, PUF. 
PETERSON J. (2014), Playing at the World: A history of simulating wars, people and fantastic adventures from chess to role-playing games [2012], San Diego, Unreason Press.

PEYRON D. (2008), « Quand les œuvres deviennent des mondes », Réseaux, 2, 148-149, pp. 335-368.

SHULGA T. (2003), « Présence médiatisée et construction de l'espace d'interaction. Comparaison entre jeux de rôles classiques et MMORPG », Les cahiers du numérique, 2, Vol. 4, pp. 101-115.

\section{NOTES}

1. La terminologie jeu de rôle papier et jeu de rôle de table désignent le même jeu et peuvent se substituer l'un à l'autre. Il est possible d'abréger ces termes par l'acronyme JdR.

2. Les jeux de rôle de table sont des « jeux de genre » qui trouvent souvent leur inspiration dans la littérature (e.g. J. R. R. Tolkien, F. Herbert, H. P. Lovecraft), les mythologies (e.g. scandinaves, germaniques), le cinéma (e.g. Star Wars, Star Trek), et plus généralement dans ce qui compose la culture populaire (Peyron, 2008 ; Peterson, 2014).

3. Nous préférons le terme de canevas à celui de scénario qui est " galvaudé par la manière dont le cinéma l'a popularisé. [...]. Quand on parle de scénario, dans l'imaginaire commun, on imagine qu'il y a un script, [...] et donc ça induit, par l'utilisation du mot scénario, quelque chose de linéaire [...]. » (de Canteloube, 2015).

4. Ces univers proposent des cadres virtuels d'interaction qui ne sont pas persistants et qui sont par conséquent nécessairement réactualisés constamment à chaque partie puisqu'ils n'ont pour seul fondement en jeu que les représentations imaginaires des joueurs, leur récit collectif et leurs actions virtuelles (Shulga, 2003).

5. Nous entendons par dispositif toute solution logicielle ou matérielle participant à l'augmentation ou l'amélioration de l'expérience de jeu des rôlistes, tandis que nous entendons par ressource toute production finale directement exploitable par les rôlistes pour augmenter ou améliorer leur expérience de jeu.

6. Le roleplay correspond à l'interprétation immersive et créative que fait un joueur de son personnage. Cette interprétation doit non seulement se faire dans un souci de réalisme et de cohérence intradiégétique, mais également dans un but de renforcement et d'enrichissement de la réalité fictionnelle dans laquelle elle s'inscrit.

7. Le jeu de rôle textuel dont il est question ici n'est pas à confondre avec les MUD.

\section{RÉSUMÉS}

Cet article s'intéresse à l'évolution de la pratique du jeu de rôle à l'ère du numérique, principalement en France. Internet, le Web 2.0, et les autres dispositifs numériques sont pratiquement omniprésents dans notre société moderne. Les jeux, notamment les jeux de rôle, ne sont pas épargnés par cette imprégnation numérique puisque l'expérience de jeu des rôlistes est bien souvent augmentée et améliorée à travers ces nouveaux dispositifs. Ce travail se propose d'observer les nouveaux comportements et usages des rôlistes français dans leur pratique du jeu de rôle à l'ère du numérique grâce à un questionnaire soumis à de nombreux sujets (rôlistes). 
D'autre part, avec l'intégration des nouvelles technologies dans les univers ludiques, se pose la question de l'intégrité de l'essence du jeu de rôle dont les frontières sont souvent redéfinies.

This article focuses on the evolution of the practice of roleplaying games in the digital era. Internet, the Web 2.0, and other digital devices are nearly omnipresent in our modern society. Games, including roleplaying games, are not spared by this digital impregnation since the gaming experience of the role players is often enhanced and improved through these new devices. This work observed the new behaviours and uses of french role players using a questionnaire submitted to numerous subjects. Furthermore, the introduction of new technologies into playful sphere raises the issue of the role playing games wholeness for which the boundaries are often redefined.

\section{INDEX}

Mots-clés : jeux de rôle, jeu de rôle papier, jeu de rôle de table, ère numérique, usages, comportements, dispositifs numériques

Keywords : roleplaying game, pen and paper roleplaying game, tabletop roleplaying game, digital era, uses, behaviors, digital devices

\section{AUTEUR}

\section{UGO ROUX}

Université de Toulon, I3M 